\title{
On the artificial creation of the EL2 center by means of boron implantation in gallium arsenide
}

\author{
J. R. Morante, A. Perrez-Rodríguez, J. Samitier, and A. Romano-Rodríguez \\ L. C. M. M., Departament de Fisica Aplicada i Electrònica, Universitat de Barcelona, \\ Avda. Diagonal 645-647, 08028-Barcelona, Spain
}

(Received 25 April 1991; accepted for publication 25 June 1991)

\begin{abstract}
In the present work, an analysis of the dark and optical capacitance transients obtained from Schottky Au:GaAs barriers implanted with boron has been carried out by means of the isothermal transient spectroscopy (ITS) and differential and optical ITS techniques. Unlike deep level transient spectroscopy, the use of these techniques allows one to easily distinguish contributions to the transients different from those of the usual deep trap emission kinetics. The results obtained show the artificial creation of the EL2, EL6, and EL5 defects by the boron implantation process. Moreover, the interaction mechanism between the EL2 and other defects, which gives rise to the $U$ band, has been analyzed. The existence of a reorganization process of the defects involved has been observed, which prevents the interaction as the temperature increases. The activation energy of this process has been found to be dependent on the temperature of the annealing treatment after implantation, with values of 0.51 and $0.26 \mathrm{eV}$ for the as-implanted and $400^{\circ} \mathrm{C}$ annealed samples, respectively. The analysis of the optical data has corroborated the existence of such interactions involving all the observed defects that affect their optical parameters.
\end{abstract}

\section{INTRODUCTION}

EL2 constitutes one of the more interesting defects in GaAs. ${ }^{1,2}$ From the technological point of view, it plays a significant role in the availability of semi-insulating substrates, this being fundamental to GaAs integrated circuit (IC) technology. Physically, this defect presents a complex behavior which is characterized by the existence of an optical property, the photoquenching effect. This effect is used to identify the presence of the defect. It is based on the existence of a metastable state of the optically inactive center, which is observed when the sample is illuminated with photons in the energy range $0.9-1.4 \mathrm{eV}$ at low temperatures (below $120 \mathrm{~K}$ ).

These features have caused a strong interest in the investigation of the nature of the EL2 center. In spite of the high number of works devoted to the characterization of the defect, its microscopic nature is still not well known. There is, however, experimental evidence which indicates that this defect has a complex structure that involves the arsenic antisite, $\mathrm{As}_{\mathrm{Ga}}$ and surrounding sites. ${ }^{3,4}$

In this framework, the study of the artificial creation of the EL2 center by means of irradiation or implantation processes has a strong interest. This allows one to obtain significant information that enables a better understanding of the nature of EL2. Since Jervis, Woodard, and Eastman ${ }^{5}$ reported their data on the artificial creation of a level, likely EL2, by Si implantation in GaAs, many papers have been devoted to the possible artificial creation of this center. $^{6-10}$ In these works, the analysis of the defects present in the samples was done by standard electrical techniques, such as DLTS (deep level transient spectroscopy) ${ }^{11}$ or DLOS (deep level optical spectroscopy). ${ }^{12}$ The reported data show the existence of differences in the Arrhenius activation energies of the elec- tron emission rate and in the optical quenching spectra. These differences are related to the characteristics of the starting material, the implantation or irradiation process, and the annealing treatment. This behavior points out the complex nature of the EL2 defect and has led to the concept of an EL2 family. ${ }^{13}$ According to this model, EL2 does not correspond to a unique defect, and its characteristics depend on the presence and nature of other defects surrounding the center (already present in the starting material or induced during its processing). ${ }^{14}$

Moreover, the DLTS spectra obtained from these samples are characterized by the presence of a broad peak at temperatures lower than that corresponding to the EL2 one. This peak, labelled the $U$ band, corresponds to nonexponential transients. Its evolution with annealing temperature suggests the existence of a direct relationship with the EL2 peak. In order to explain this behavior, different authors have assumed the existence of an interaction mechanism between the EL2 and a shallower level, identified as EL6. ${ }^{10,15,16}$ A similar mechanism has been suggested by Makram-Ebeid and Boher, ${ }^{17}$ for the case of electron-irradiated samples. However, the dependence of the amplitude and shape of the $U$ band on the sampling time used in the DLTS measurement reveals the presence of problems in the interpretation of the spectra, because DLTS is not well suited for the analysis of these mechanisms.

In this paper, in order to clarify the existence of interaction mechanisms related to EL2 and their relationship with the artificial creation of this center, a systematic study of the defects introduced by boron implantation in $n$-type Bridgman GaAs is reported. The interest in boron is due to the fact that this element is often a residual isoelectronic impurity in the gallium arsenide lattice. It has been reported to produce complexes with a reduction of the freeelectron concentration similar to that observed for 


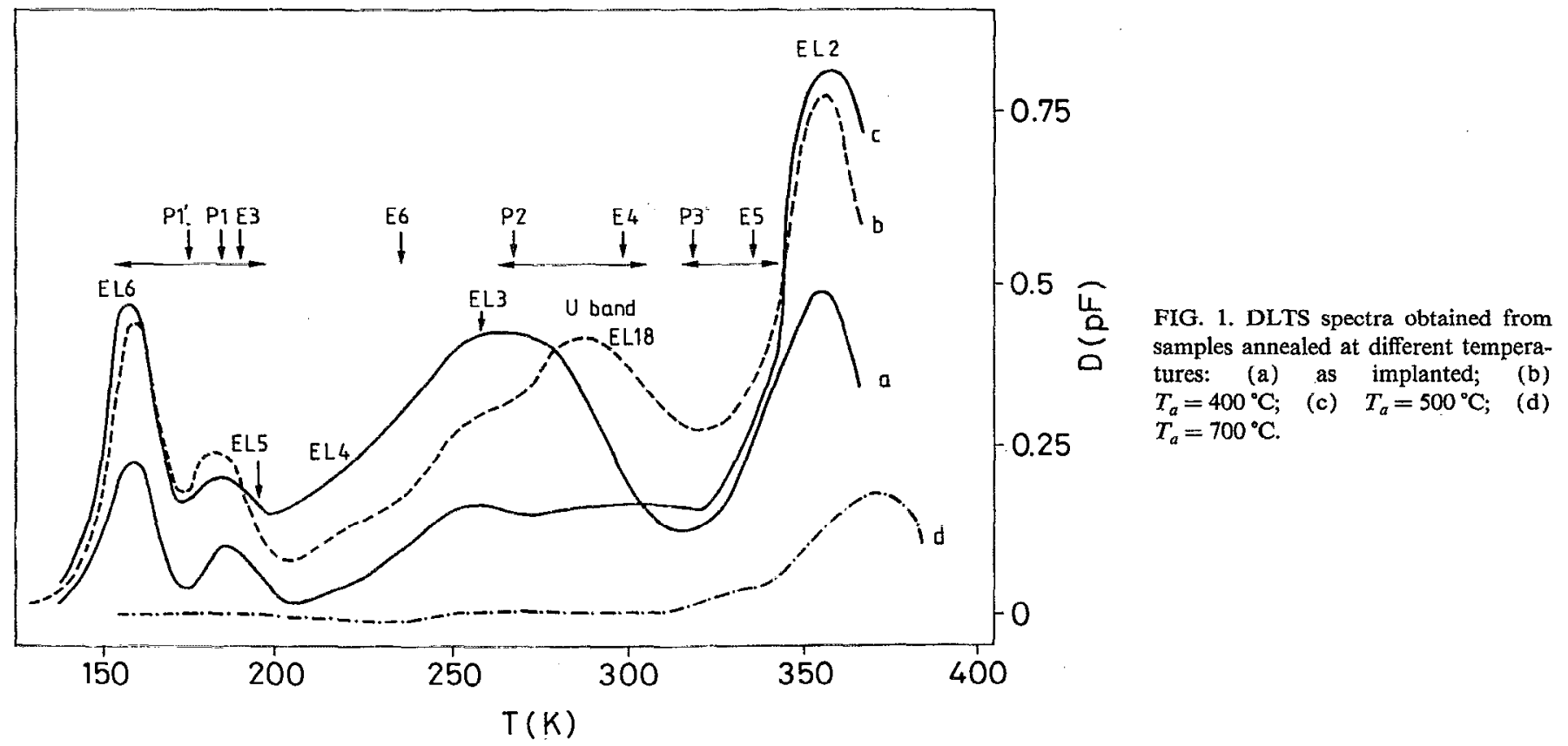

oxygen, ${ }^{18}$ but with less lattice damage. This has interest for the creation of electrical isolation in integrated circuits and optoelectronic devices.

In order to avoid the problems that appear when DLTS is applied to complex systems (systems with nonexponential behavior or with the presence of more complex phenomena than the direct emission to the bands in the emission process), we have used an experimental technique that is based on the analysis of the isothermal transients, the dark and optical isothermal transient spectroscopies (ITS and OITS techniques). ${ }^{19}$

\section{SAMPLES}

Boron has sometimes been observed in a large concentration in liquid-encapsulated-Czochralski (LEC) material. Then, to avoid possible effects of residual boron contamination, we have selected as starting material horizontal Bridgman $n$-GaAs, Te doped with a free-electron concentration of about $8 \times 10^{16} \mathrm{~cm}^{-3}$. Before the implantation process, and in order to decrease the native electron trap concentration in a $1-\mu \mathrm{m}$-thick layer below the surface, samples were capped with a $1000-\AA$ chemical-vapor-deposited (CVD) $\mathrm{Si}_{3} \mathrm{~N}_{4}$ layer and annealed at $870^{\circ} \mathrm{C}$ for $15 \mathrm{~min}$. After removing the $\mathrm{Si}_{3} \mathrm{~N}_{4}$ layer, the samples were implanted with boron except for the control one. In all the cases, the dose was $10^{11}$ ions $/ \mathrm{cm}^{2}$ and the ion energy was $100 \mathrm{keV}$. As previously reported ${ }^{20}$ layers implanted with this dose show a maximum of resistivity. Thus, for this dose a maximum of the effects and mechanisms of compensation due to the implantation process, and related to the defects induced by the process, are to be expected. After the implantation, annealing treatments were carried out in an open furnace, using the close-contact technique under a $\mathrm{H}_{2}$ flux for $15 \mathrm{~min}$. The annealing temperature was in the range between 300 and $700^{\circ} \mathrm{C}$. Finally, Schottky diodes were obtained by evaporating gold electrodes on the surface.

\section{EXPERIMENTAL TECHNIQUE: ITS AND OITS TECHNIQUES}

The characterization of the samples by the DLTS technique has allowed us to corroborate the existence of a nonexponential behavior in the capacitance transients, associated with the $U$ band. The DLTS spectra also show the presence of other peaks with an exponential shape (Fig. 1) ${ }^{21}$ In general, a nonexponential behavior can appear: (i) when the trap density is large compared to the free-carrier concentration, (ii) when the edge region width is not negligible, (iii) when the emission rate is electric-field dependent, (iv) due to the contribution of different exponential transients, with closely spaced time constants, or, finally, (v) due to a more complex behavior of the emission kinetics as, for example, the existence of interaction processes between defects which, in general, will be temperature dependent.

In our case, the presence in the DLTS spectra of exponential peaks suggests the origin of the nonexponential behavior to be related to the intrinsic characteristics of the defects involved. In such a case, the analysis by DLTS of the capacitance transients has problems and may lead to gross misinterpretation. So, DLTS assumes an exponential behavior with a pre-exponential factor independent on the temperature. However, this factor can be temperature dependent, for example, due to a dependence of the defect concentration or capture time on the temperature. In these cases the analysis of the transients obtained at isothermal conditions has been reported to be more suited and accurate for the study of this behavior. ${ }^{19,22}$ 
Let $S(t)$ be the capacitance transient due to a deep level and obtained when the polarity changes from forward to reverse bias in darkness or under optical excitation. This transient can be written in terms of a general time constant distribution function $G(v)$,

$$
S(t)=A\left(1-\int_{0}^{\infty} G(v) \exp (-v t) d v\right),
$$

where $v=1 / \tau$ and $A$ is proportional to the ratio of the deep trap to shallow level concentrations $N_{T} / N_{D}$. In the case of a level interacting with the bands via ShockleyRead and Hall statistics the transient is exponential. In this case $G(v)$ is given by a Dirac function, $G(v)=\delta(v$ $-v_{0}$ ), where $v_{0}$ is the total emission coefficient of the level.

The ITS signal of a general transient $S(t)$ is defined as

$$
Y(t)=\frac{d S(t)}{d(\ln t)}=\lim _{k \rightarrow 1} \frac{\left[S\left(t k^{1 / 2}\right)-S\left(t k^{-1 / 2}\right)\right]}{\ln k} .
$$

Making the change of variables $x=\ln (t), y=-\ln (v)$, the ITS signal is deduced to be given by

$$
Y(x)=\int_{-\infty}^{\infty} \bar{G}(y) h(x-y) d y,
$$

where the $h(x)$ and $\bar{G}(y)$ functions are defined as

$$
\begin{aligned}
& h(x)=\exp [x-\exp (x)], \\
& \bar{G}(y)=\exp (-y) G[\exp (-y)] .
\end{aligned}
$$

So, the ITS signal is given by a convolution product, where $h(x)$ corresponds to the $Y(x)$ signal of an exponential transient. The shape of the ITS spectrum will be similar to that of the $\bar{G}(y)$ function, modulated by the fact that the $h(x)$ function is not a Dirac function. The experimental implementation of this method is easier than that of the methods based on the Fourier transform ${ }^{23,24}$ or the method of moments. ${ }^{25}$

Therefore, the ITS spectra allow us to characterize a general transient. For an exponential transient, the ITS spectrum has a characteristic shape: It exhibits a maximum located at $t=1 / v_{0}$ with an amplitude given by $A \exp (-1)$, and a full width at half-height of 1.06 decades (for $k \rightarrow 1$ ). The numerical differentiation of Eq. (1) by expression (2) using a value of $k>1$ improves the signalto-noise performance; however, it increases the width of the peak. The optimization of this parameter $(1<k<2)$ allows one to separate time constants up to a ratio of 3 with an optimum signal-to-noise ratio, and it is also possible to separate the exponential and nonexponential contributions of the different levels to the transient. ${ }^{19}$ Moreover, the analysis of the differential ITS (DITS) spectra allows the study of the dependence of the emission kinetics on the electric field to be made. 22

\section{EXPERIMENTAL RESULTS}

In order to characterize the samples, we have carried out capacitance transient measurements in darkness and under optical excitation conditions. The transients were
ITS

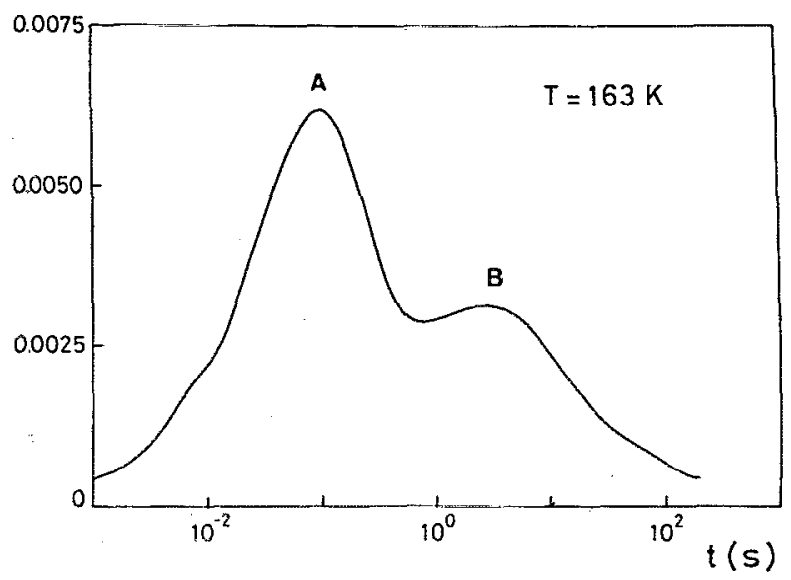

FIG. 2. ITS spectrum from the as-implanted sample at $T=163 \mathrm{~K}$, showing two exponential peaks labeled $\mathbf{A}$ and $\mathbf{B}$.

measured from $10^{-4}$ to $10^{3} \mathrm{~s}$. The lower limit is determined by the experimental system of measurement of the capacitance, and the upper one is used in order to minimize thermal drift effects.

\section{A. Dark capacitance transients}

The ITS spectra obtained from transients measured in darkness at different temperatures show 3 peaks of quasiexponential shape, labeled A, B, and C (Figs. 2 and 3 ). Moreover, there also appears a broad nonexponential band. This band corresponds to the $\mathrm{U}$ band found by previous DLTS measurements ${ }^{21}$ (Fig. 3). From an Arrhenius plot of these peaks, we have deduced the values of the activation energy and effective thermal cross section of the related levels, which are indicated in Table I. For the U

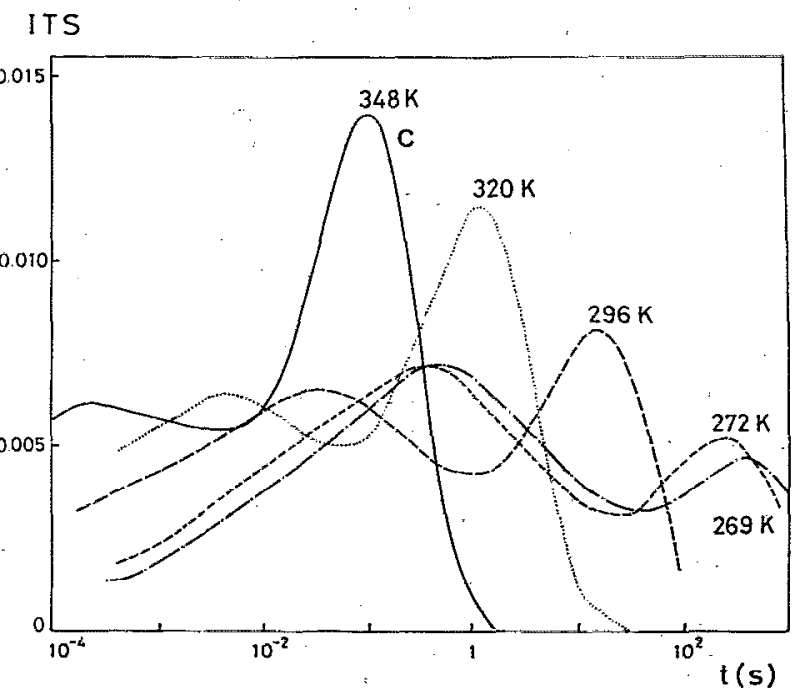

FIG. 3. ITS spectra obtained from the sample annealed at $400^{\circ} \mathrm{C}$ at different temperatures. The spectra show the exponential $\mathrm{C}$ peak and a nonexponential peak (corresponding to the $\mathrm{U}$ band). 
TABLE I. Signatures of the A, B, and C peaks observed in the ITS spectra.

\begin{tabular}{lcc}
\hline \hline Peak & $\begin{array}{c}\text { Activation energy } \\
(\mathrm{eV})\end{array}$ & $\begin{array}{c}\text { Effective thermal cross } \\
\text { section } \sigma_{\infty}\left(\times 10^{-14} \mathrm{~cm}^{2}\right)\end{array}$ \\
\hline A & $0.345 \pm 0.01$ & 8 \\
B & $0.40 \pm 0.01$ & 12 \\
C & $0.79 \pm 0.02$ & 7.4 \\
\hline
\end{tabular}

band, we have also observed the existence of a thermalactivated shift of the band. However, its activation energy has been found to depend on the annealing temperature of the sample, with values of 0.49 and $0.67 \mathrm{eV}$ for the asimplanted and $400^{\circ} \mathrm{C}$ anncaled samples, respectively.

According to the values shown in Table I, A and C peaks have been identified as the EL6 and EL2 levels, respectively. ${ }^{26,27}$ For the $B$ peak, its parameters agree with those reported for the levels of the E3 family. This corresponds to an ensemble of levels whose reported signatures are very similar to those of E3, as EL5, P1, or P1' defects. ${ }^{26,28-31}$ Then, in order to make the complete identification of this defect, we have also to take into account the dependence of the peak with the electric field, as well as its optical behavior. These are reported in the next sections.

The evolution of the spectra with the temperature of measurement is as follows:

(i) At temperatures below $200 \mathrm{~K}$, the spectra only show the $A$ and $B$ peaks (Fig. 2).

(ii) At temperatures higher than $200 \mathrm{~K}$, the $\mathrm{U}$ band appears.

(iii) At temperatures higher than $260 \mathrm{~K}$, the spectra only show the presence of both the $U$ band and the EL2
(C) peaks (Fig. 3). The amplitude of both peaks depends on the temperature: As the temperature increases, the amplitude of the $U$ band decreases and the amplitude of the EL2 peak increases. However, the total emitted charge remains constant within our experimental accuracy. This fact suggests the existence of an interaction mechanism related to the EL2 center.

On the other hand, the evolution of the spectra with the annealing temperature of the samples $T_{a}$ is similar to that observed for the temperature of measurement $T$ : At a given $T$, as $T_{a}$ increases the amplitude of the EL2 peak increases in relation to that of the $U$ band (Fig. 4). For annealing temperatures higher than $500^{\circ} \mathrm{C}$ only the EL2 peak appears.

The dependence of these peaks on the applied electric field has been studied by means of the DITS technique. For the A peak, the observed dependence is similar to that reported for the EL6, ${ }^{32}$ and corroborates its identification with this center. For the B peak, the dependence of time constant on the electric field is smaller than that reported in the literature for the E3 level (Fig. 5).$^{33}$ This fact indicates that the $B$ peak corresponds to another level from the E3 family. Finally, we have not observed any dependence of the position of the EL2 peak on the electric field. This has been due to the fact that the values used for the electric field were lower than the threshold value for this level. ${ }^{34}$

\section{B. Optical capacitance transients}

In order to complete the identification of the defects, as well as to clarify. the question about the origin of the $U$

\section{ITS (a.u.)}

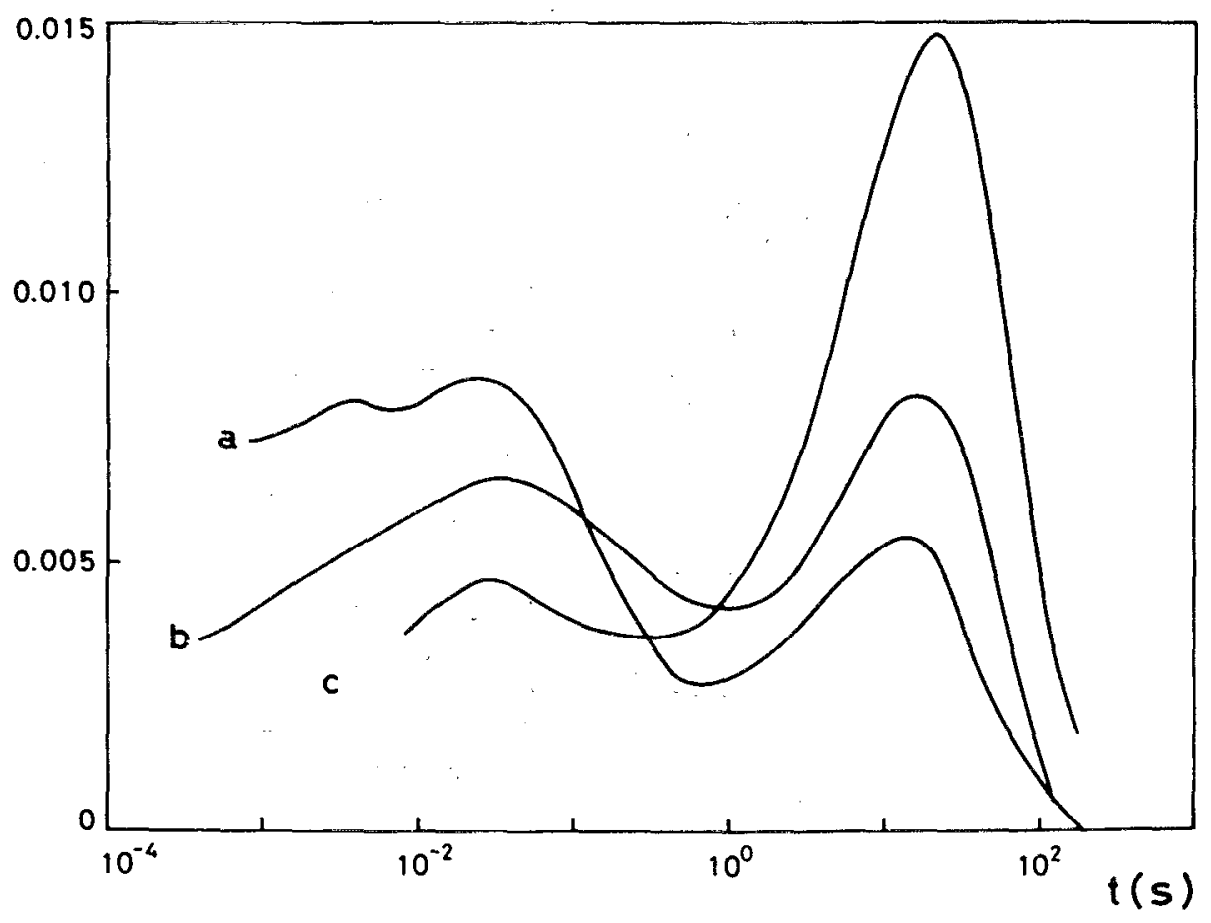

FIG. 4. Normalized ITS spectra from samples (a) as implanted, (b) annealed at $400^{\circ} \mathrm{C}$, and (c) annealed at $475^{\circ} \mathrm{C} . T=292$ $\mathbf{K}$. 


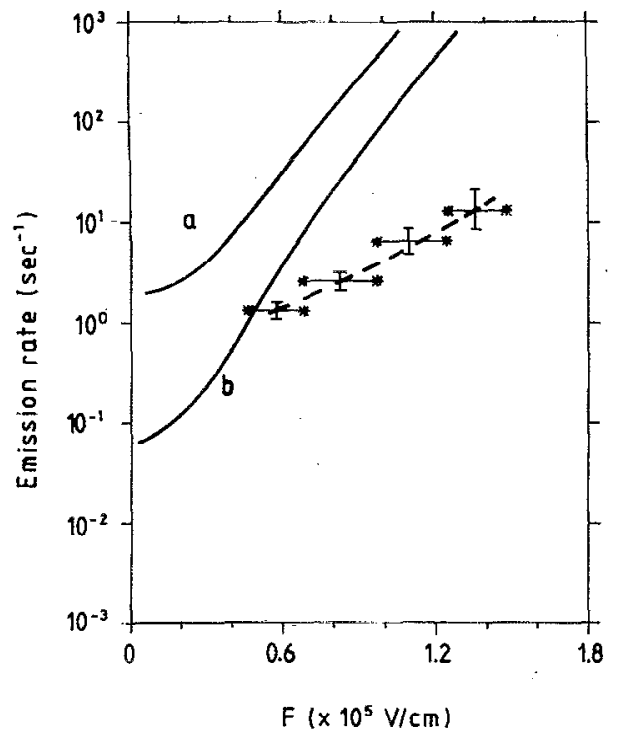

FIG. 5. Electric-field dependence of the emission coefficient from B level at $T=174 \mathrm{~K}$. Solid lines correspond to the reported for E3 at (a) $T=180 \mathrm{~K}$ and (b) $T=160 \mathrm{~K}$ (Ref. 33 ).

band and its relationship with the EL2, we have carried out transient measurements under different optical and initial conditions.

In Fig. 6 are plotted the OITS spectra corresponding to the phototransients obtained at $284.2 \mathrm{~K}$ and an illumination energy of $1.3 \mathrm{eV}$ for different intensities of illumination. As is shown, at the higher illumination intensity a peak of exponential shape is obtained whose characteristics are similar to those reported for the EL2 level. As the illumination intensity decreases the time constant related to the photoionization of this level increases. Then, the broad band corresponding to the thermal emission of the $U$ band appears. These results suggest that the $U$ band does

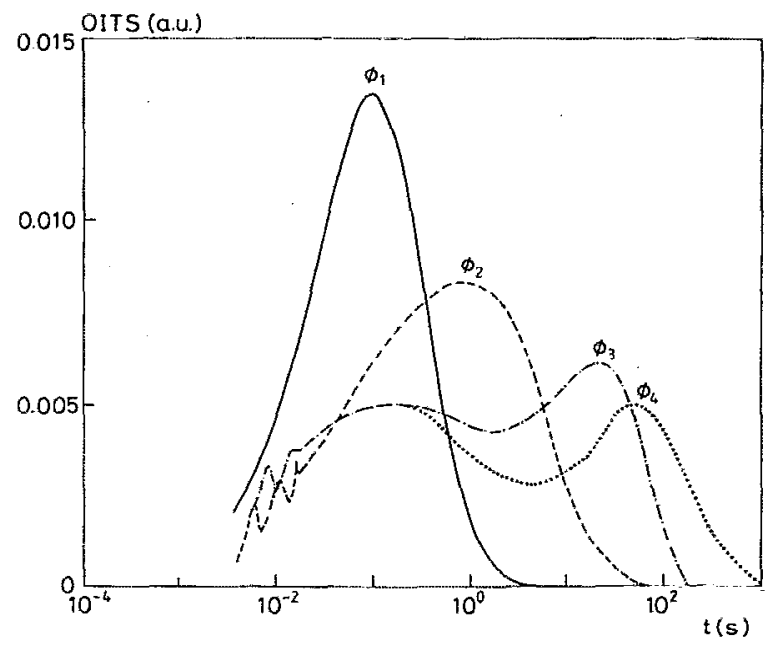

FIG. 6. Normalized OITS spectra obtained from the sample annealed at $400^{\circ} \mathrm{C}$ with an illumination cnergy of $1.3 \mathrm{eV}$ and different intensitics: $\phi_{1}>\phi_{2}>\phi_{3}>\phi_{4}=0 ; T=284.2 \mathrm{~K}$.
OITS

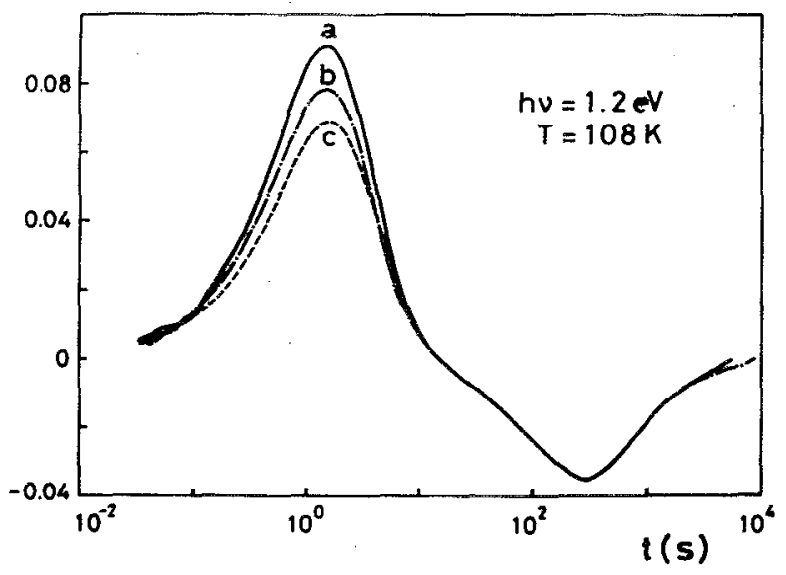

FIG. 7. OITS spectra obtained for different values of the occupancy factor of A and B levels: (a) A and B full of electrons; (b) A empty and B full; and (c) $A$ and $B$ empty.

not have an optical response. Under illumination, the charge which in darkness is thermally emitted from the $U$ band is directly photoionized from the EL2 defect when the probability of this last process is larger than that of thermal emission from the $U$ band.

The optical response of the different levels has been determined by the analysis of the optical transients obtained at low temperature (about $100 \mathrm{~K}$ ) and with different values of the occupancy factor of the $A$ and $B$ levels. This has been achieved by changing the polarity of the diode from forward to reverse bias before illuminating at higher temperature (about $140 \mathrm{~K}$ ), and maintaining this temperature for a time long enough to obtain the thermal emission of the A or both A and B levels. In this way, we have obtained different spectra corresponding to the transients measured with different initial conditions: (i) all the levels full of electrons, (ii) A level empty, and (iii) both A and $B$ levels empty (Fig. 7).

The spectra obtained under these conditions and for energies higher than $0.9 \mathrm{eV}$ show a positive and a negative peak, independently of the occupancy factor of A and B levels. Both peaks have a quasiexponential shape. The negative peak indicates the presence in the transients of an exponential component that decreases in time. This corresponds to the optical quenching associated with the EL2 level, and gives evidence of the presence of this level from the optical point of view. The amplitude of the positive OITS peak depends on the occupancy factor of A and B levels, but its position and the amplitude and position of the negative peak depend only on the photon energy.

The OITS spectra corresponding to the contribution of A and B levels to the optical transients have been obtained by subtracting the spectra obtained with different occupancy factors. They show a quasiexponential behavior. However, for the higher illumination energies they have a width smaller than that corresponding to the exponential case (Fig. 8). As discussed later, this fact gives evidence of the existence of an interaction mechanism in the optical 


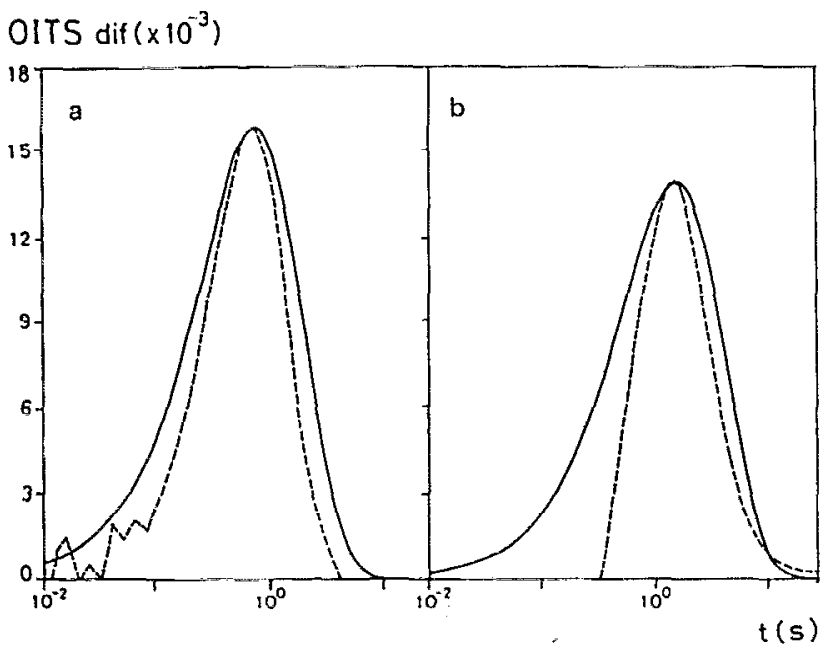

FIG. 8. Dotted lines are the differential OITS spectra from (a) A and (b) B levels at $T=108 \mathrm{~K}$ and $h v=1.3 \mathrm{cV}$. Solid lines correspond to the OITS signal of an exponential transient.

transients between these defects and the EL2. Moreover, the area of these differential peaks does not depend on the photon energy. This indicates that both levels have a négligible optical hole cross section. This behavior points out that these levels have a high value of their Frank-Condon shift $d_{\mathrm{FC}}$, and has allowed us to complete the identification of the defects, as indicated in Table II. So, for the A peak this behavior corroborates its identification with the EL6 level, characterized by a high value of $d_{\mathrm{FC}}$. The level related to the $B$ peak can be identified as the EL 5 one, which is the defect from the E3 family that exhibits such behavior.

Finally, for the transients obtained with $\mathrm{A}$ and $\mathrm{B}$ levels previously emptied one should expect to obtain only the optical response of the EL2 level. Then, the ratio between the positive and the negative peak amplitude should be unity. However, this is only true for photon energies smaller than $1.1 \mathrm{eV}$. For higher energies, this ratio increases greatly.

\section{DISCUSSION}

It has to be remarked that, taking into account the values used to bias the samples, the region studied has always been enclosed in the 1- $\mu \mathrm{m}$-thick surface layer. In

TABLE II. Identification of the different peaks, together with their Frank-Condon shift $d_{\mathrm{FC}}$.

\begin{tabular}{ccc}
\hline Peak & Defect & $\begin{array}{c}d_{\mathrm{FC}} \\
(\mathrm{eV})\end{array}$ \\
\hline A & EL6 & $0.6^{\mathrm{a}}$ \\
B & EL5 & $0.48^{\mathrm{b}}$ \\
C & EL2 & $0.12^{\mathrm{c}}$ \\
\hline
\end{tabular}

See Ref. 35.

SSee Ref. 36.

'See Ref. 12. this region native traps were removed before implantation. So, all the defects observed correspond to defects created during the different processing steps. The optical results show the presence of EL2 already after implantation (without annealing), which gives evidence of the artificial creation of this center by the implantation process.

On the other hand, the relationship found between the U-band and the EL2 peaks in the dark transients corroborates the existence of an interaction mechanism related to the EL2 level, as reported by different authors. ${ }^{10,15-17}$ According to these works, the emission of the electrons from the EL2 level can take place directly to the conduction band (giving rise to the exponential peak $\mathrm{C}$ ) or through another defect previously ionized, likely via a hopping mechanism thermally activated. In such a case, as the probability of transition of the electrons from the EL2 to the other defect depends on the distance between them, a broad band ( $U$ band) corresponding to a time constant distribution will appear.

Moreover, the evolution of the amplitude of the EL2 peak and the $U$ band with the temperature indicates that a certain portion of the EL2 defect does not interact with the other defect. This portion increases with the temperature. This can be cxplained assuming the existence of a reorganization process of the involved defects which rules out the interaction. Taking into account the high stability of the EL2 center under thermal treatments, we have assumed that the reorganization process corresponds to a dissociation of the complex partner of the EL2 in the interaction

$$
\mathrm{EL} 2+(X Y) \rightleftharpoons \mathrm{EL} 2+X+Y \text {. }
$$

The evaluation of the equilibrium constant of this reaction from the experimental spectra has allowed us to deduce this process to be thermally activated (Fig. 9). The activation energy of this process diminishes as the annealing temperature increases. The values estimated for the as-implanted and the $400^{\circ} \mathrm{C}$-annealed samples are 0.51 and 0.26 $\mathrm{eV}$, respectively. This evolution suggests this process to be dependent on the presence of other defects surrounding the involved centers. So, the probability of dissociation increases as the concentration of these defects decreases by thermal annealing. Moreover, the behavior found for the different annealing temperatures indicates that this dissociation is a reversible process for temperatures below the maximum temperature of measurement $(348 \mathrm{~K})$. However, for higher temperatures (of the order of the temperatures used in the annealing processes, higher than $300^{\circ} \mathrm{C}$ ) it becomes irreversible.

As the other defects found, EL6 and EL5, are shallower than EL2, and in agreement with the previous works reported, we have assumed that the EL2 is interacting with a shallower defect. The time constant distribution that appears from this interaction process can be described taking into account the following features: (i) the probability of a thermally activated hopping transition between the defects, given by

$$
e_{t}=e_{0} \exp (-E / k T) \exp (-2 r / \Gamma)
$$




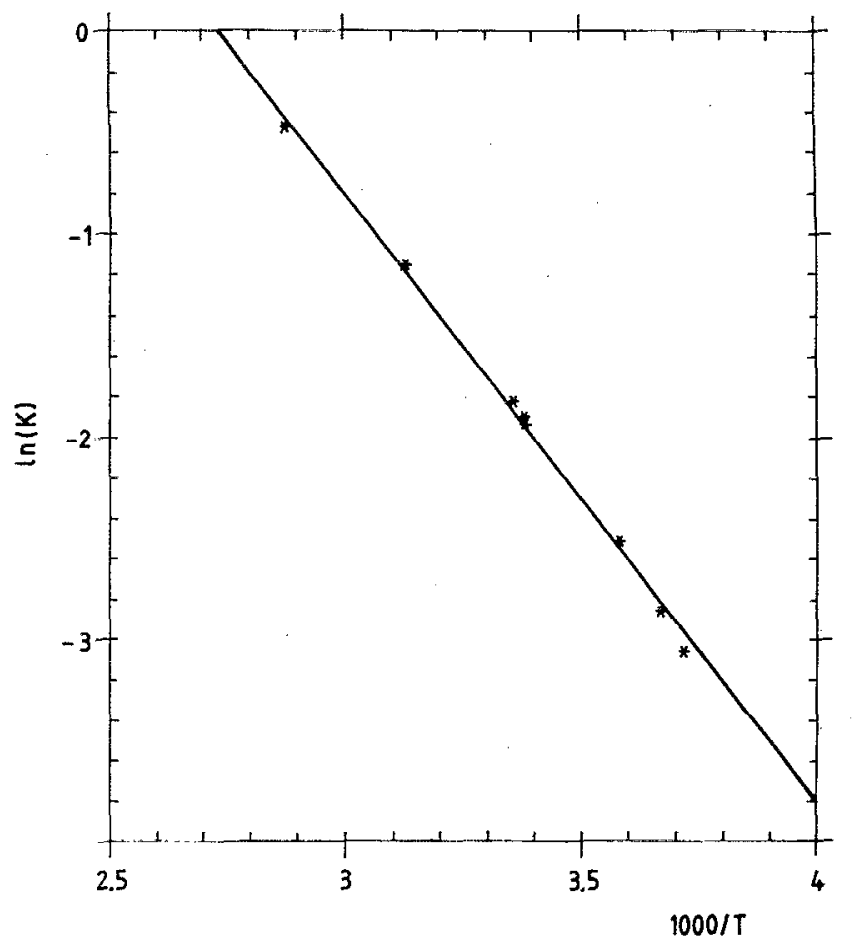

FIG. 9. Arrhenius plot of the equilibrium constant associated with the reorganization process from the sample annealed at $400^{\circ} \mathrm{C}$.

where $E$ is the barrier height between both localized defects, $r$ is the distance between them, and $\Gamma$ is a parameter related to their wave function overlapping, ${ }^{37}$ and (ii) the existence of a distance distribution between both defects, which has been considered to be like a donor-acceptor-pair distribution.

From these assumptions, straightforward calculations allow us to deduce the following expression for the time constant distribution of the emission process from the EL2 defect:

$$
G(\tau)=\frac{\pi}{2} N \Gamma^{3} h^{2}(\tau) \exp \left(-\frac{\pi}{6} N \Gamma^{3} h^{3}(\tau)\right) \frac{\tau_{2}}{\tau\left(\tau_{2}-\tau\right)},
$$

$N$ is the concentration of the defect interacting with the EL2, $\tau_{2}$ is the time constant related to the direct emission of the EL2 to the conduction band (given by its emission coefficient), and $h(\tau)$ is

$$
h(\tau)=\ln \frac{\tau \tau_{2}}{\left(\tau_{2}-\tau\right) \tau_{0} \exp (E / k T)},
$$

where $\tau_{0}=1 / e_{0}$.

From expressions (2) to (4) it is possible to calculate the ITS signal related to this process. This model allows us to simulate, in a qualitative way, the broad time constant distribution related to the $U$ band if we consider a Gaussian profile distribution of the defects, related to the implantation process. Taking into account the thermal activated reorganization process of the defects involved in the interaction it is possible to obtain the sharp increase of the amplitude of the EL2 peak and the decrease of that of the

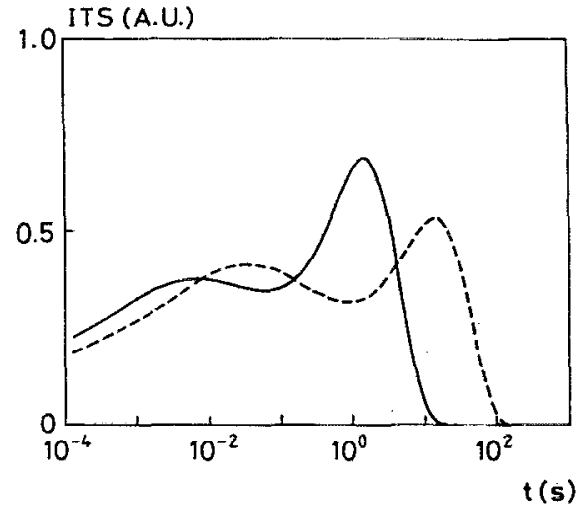

FIG. 10. Theoretical spectrum obtained at $T=298 \mathrm{~K}$ (dotted line) and $T^{\prime}=320 \mathrm{~K}$ (solid line), assuming a Gaussian profile of the defects with concentrations between $5 \times 10^{15}$ and $5 \times 10^{17} \mathrm{~cm}^{-3}$.

$\mathrm{U}$ band as the temperature increases, reproducing the experimental spectra (Fig. 10).

From these measurements it is not possible to determine which is the defect interacting with the EL2. However, the optical measurements carried out give experimental evidence of both EL6 and EL5 defects interacting with the EL2. So, the characteristics of the positive peak of the OITS spectra indicate two possibilities: (i) The optical time constant of these levels is very similar to that of the EL2, or (ii) there is an interaction process between the EL2 and these defects, and the photoionization of the EL6 and EL5 defects occurs through the EL2, previously ionized. However, the differential OITS peaks corresponding to the optical response of the EL6 and EL5 defects show for the higher illumination energies a width smaller than that of an exponential peak (Fig. 8). Such a behavior cannot be explained with the first possibility. On the contrary, it can be simulated assuming the existence of an interaction between the defects. ${ }^{38}$ In order to account for this interaction we have assumed the following expressions:

$$
\begin{aligned}
& \frac{d n_{1}}{d t}=e_{p 1}^{o}\left(N_{1}-n_{1}-n_{1}^{*}\right)-e_{n 1}^{o} n_{1}-e^{o *} n_{1} \\
& \quad+\alpha n_{2}\left(N_{1}-n_{1}-n_{1}^{*}\right), \\
& \frac{d n_{1}^{*}}{d t}=e^{o *} n_{1}, \\
& \frac{d n_{2}}{d t}=e_{p 2}^{o}\left(N_{2}-n_{2}\right)-e_{n 2}^{o} n_{2}-\alpha n_{2}\left(N_{1}-n_{1}-n_{1}^{*}\right) .
\end{aligned}
$$

$N_{1}$ is the total concentration of the EL2 level, and $n_{1}$ and $n_{1}^{*}$ are the electron concentrations in its fundamental and metastable states, respectively. $N_{2}$ and $n_{2}$ are the total and electron concentrations of the level interacting with the $\mathrm{EL2}, e_{n i}^{o}$ and $e_{p i}^{o}$ are the optical emission coefficients of the electrons and holes in the different levels, and $e^{o *}$ is the probability of transition of electrons from the fundamental to the metastable state of the EL2. Finally, $\alpha$ represents the probability of transition of electrons from the defect inter- 


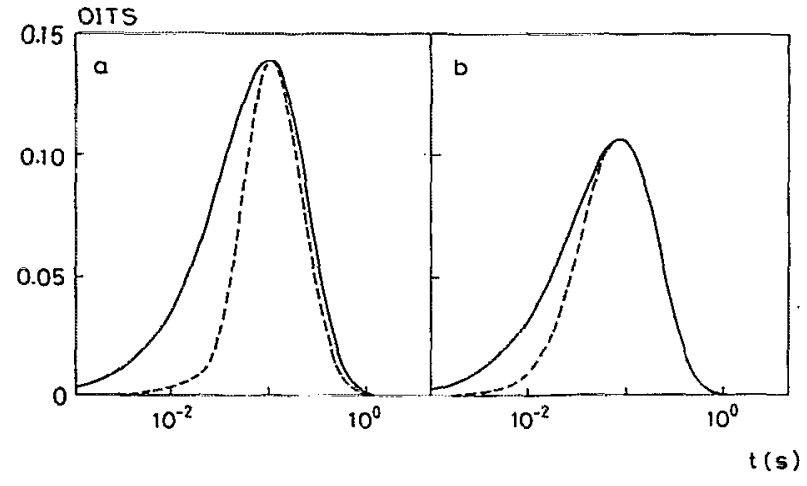

FIG. 11. Dotted lines are the theoretical OITS differential spectra for (a) $A$ and (b) B levels. Solid lines correspond to the OITS signal of an exponential transient.

acting with EL2 to the EL2 level, by an interaction mechanism similar to that mentioned above.

In Figs. 11(a) and 11(b) are plotted the theoretical differential spectra obtained from the generalization of Eqs. (8) for two levels (A and B) interacting with the EL2. In these figures we have considered for the different parameters the values corresponding to the EL6, EL5, and EL2 centers. If the probability of transition $\alpha$ is high enough when compared to the photoionization probability of the A and B defects, the photoemission of the electrons in these centers takcs place essentially through the EL2, previously ionized. As it is shown in these figures, in such a case it is possible to simulate the observed behavior. The dependence of the full width at half-height of the experimental spectra with the illumination energy suggests an enhancement of this interaction as the illumination energy increases.

Finally, we have already indicated how the OITS spectra obtained with both A and B levels previously emptied show an increase of the amplitude of the positive peak in relation to that of the negative one as the illumination energy increases. In this case, only the optical response from the EL2 defect would be expected. This behavior cannot be explained from the increase of the ratio between the electron and hole optical cross sections of the EL2. Essentially, two possibilities can explain this behavior: (i) the existence of an optically induced regeneration of the metastable state of the center, as suggested by different authors ${ }^{39-42}$ and (ii) the contribution of other levels, energetically located below the midgap. In relation to the second possibility, the implantation process is expected to induce nondetected acceptor levels in relatively high concentration (in order to justify the compensation of the material by boron implantation). However, if this possibility is the correct one, the optical response of these levels should have the same time constant as the EL2, or they would also interact with the EL2 complex. Anyway, a more detailed analysis needs to be done in order to elucidate between these possibilities.

\section{SUMMARY}

In conclusion, the thermal and optical characterization of GaAs samples implanted with boron by the ITS and OITS techniques has allowed us to identify the main defects in the upper half of the gap as EL6, EL5, and EL2. These defects have been artificially created by the implantation process. Moreover, we have corroborated and analyzed the existence of an interaction related to the EL2 which gives rise to the $U$ band. On the other hand, from the measurements carried out under optical excitation we have observed how both EL6 and EL5 defects interact with the EL2. This supports the idea that one of these defects, or maybe both of them, is the defect interacting with the EL2 giving rise to the $\mathrm{U}$ band.

Likewise, the analysis performed has corroborated the ability of the ITS and OITS techniques for the characterization of systems with complex behavior. So, these techniques have been proved to be more powerful than the standard ones (DLTS, DLOS) for the study of nonexponential transients from an electrical and optical point of view, especially when more complex temperature dependencies than those related to the emission coefficients are involved in the transients. The results obtained point out the complex nature of the defects artificially created by the implantation process, as well as the strong dependence of their characteristics on the defect environment.

\section{ACKNOWLEDGMENT}

The authors are indebted to the Division de Recherche Exploratoire of the Laboratoire d'Electronique Philips (LEP, Paris) for helpful discussions and for providing the samples used in this study.

'G. M. Martin and S. Makram-Ebeid, in Deep Centers in Semiconductors, edited by S. Pantelides (Gordon and Breach, New York, 1985), p. 399.

${ }^{2}$ G. Guillot, Rev. Phys. Appl. 23, 833 (1988).

${ }^{3}$ H. C. Gatos and J. Lagowski, Mater. Res. Soc. Symp. Proc. 46, 153 (1985).

${ }^{4}$ M. Bauemler, U. Kauffmann, and J. Windscheif, Appl. Phys. Lett. 46, 781 (1985).

${ }^{5}$ T. R. Jervis, D. W. Woodard, and L. F. Eastman, Electron. Lett. 15, 621 (1979).

${ }^{6}$ T. Ikoma, M. Takikawa, and M. Taniguchi, Inst. Phys. Conf. Ser. 63, 191 (1982).

${ }^{7} \mathrm{M}$. 'Taniguchi and T. Ikoma, in Semi-Insulating III-V Materials (Evian), edited by S. Makram-Ebeid and B. Tuck (Shiva, Nantwich, 1982), p. 283.

${ }^{8}$ M. Taniguchi and T. Ikoma, Inst. Phys. Conf. Ser. 65, 65 (1983).

${ }^{9}$ G. M. Martin, P. Terriac, S. Makram-Ebeid, G. Guillot, and M. Gavand, Appl. Phys. Lett. 42, 61 (1983).

${ }^{10}$ G. M. Martin, E. Esteve, P. Langlade, and S. Makram-Ebeid, J. Appl. Phys. 56, 2655 (1984).

${ }^{11}$ D. V. Lang, J. Appl. Phys. 45, 3014 (1974).

${ }^{12}$ A. Chantre, G. Vincent, and D. Bois, Phys. Rev. B 23, 5335 (1981).

${ }^{13}$ T. Ikoma and Y. Mochizuki, Jpn. J. Appl. Phys. 24, L935 (1985).

${ }^{14}$ M. O. Mancesreh and D. W. Fischer, Phys. Rev. B 40, 11756 (1989).

${ }^{15} \mathrm{P}$. Langlade and S. Makram-Ebeid, Inst. Conf. Ser. 74, 281 (1985).

${ }^{16}$ J. Samitier, J. R. Morante, L. Giraudet, and S. Gourrier, Appl. Phys. Let. 48, 1138 (1986).

${ }^{17}$ S. Makram-Ebeid and P. Boher, Rev. Phys. Appl. 23, 847 (1988).

${ }^{18}$ W. M. Paulson, M. S. Birritella, T. H. Miers, and K. L. McLaughlin, GaAs IC Symposium, 1982, p. 166.

${ }^{19}$ J. Samitier, J. R. Morante, A. Cornet, A. Herms, P. Roura, and A. Pérez, Mater. Scien. For. 10-12, 539 (1986). 
${ }^{20}$ B. K. Meyer, D. M. Hoffmann, J. R. Niklas, and J. M. Spaeth, Phys. Rev. B 36, 1332 (1987).

${ }^{21}$ A. Pérez, A. Romano, J. Samitier, H. Altelarrea, and J. R. Morante, Eur. Mater. Res. Soc. Symp. Proc. 16, 249 (1987).

${ }^{22}$ H. Altelarrea, J. Bosch, A. Pérez, J. Samitier, and J. R. Morante, Mater. Res. Soc. Symp. Proc. 163, 785 (1990).

${ }^{23}$ S. W. Provenchar, Comput. Phys. Commun. 27, 213 (1982).

${ }^{24} \mathrm{~K}$. Tkeda and H. Takaoka, J. Appl. Phys. 54, 6031 (1983).

${ }^{25}$ I. Isenberg, R. D. Dyson, and R. Hanson, Biophys. J. 13, 1090 (1973).

${ }^{26}$ G. M. Martin, A. Mitonneau, and A. Mircea, Electron. Lett. 13, 191 (1977).

${ }^{27}$ A. Yahata, T. Kikuta, and K. Ishida, Jpn. J. Appl. Phys. 25, L133 (1986).

${ }^{28}$ A. Chantre, Ph.D. thesis, Université Scientifique et Medicale de Grenoble, 1979.

${ }^{29}$ D. Pons, Ph.D. thesis, Université de Paris VI, 1979.

${ }^{30}$ Y. Kitagawara, N. Noto, T. Takahashi, and T. Takanaka, Appl. Phys. Lett. 48, 1664 (1986).
${ }^{31}$ D. Stievenard and J. C. Bourgoin, J. Appl. Phys. 59, 743 (1986).

${ }^{32}$ T. T. Nguyen, K. L. Wang, and G. P. Li, Appl. Phys. Lett. 44, 211 (1984).

${ }^{33}$ S. Makram-Ebeid, Appl. Phys. Lett. 37, 464 (1980).

${ }^{34}$ S. Makram-Ebeid and M. Lannoo, Phys. Rev. B 25, 6406 (1982).

${ }^{35}$ A. Chantre, G. Vincent, and D. Bois, Phys. Rev. B 23, 5335 (1981).

${ }^{36} \mathrm{~F}$. Litty, Ph.D. thesis, Universite Claude Bernard-Lyon I, 1982.

${ }^{37}$ V. F. Masterov and V. A. Kharchenko, Fiz. Tekh. Poluprovoln. 19, 460 (1985).

${ }^{38}$ A. Pérez, J. Samitier, J. Esteve-Tintó, A. Romano, and J. R. Morante, Def. Diff. For. 62-63, 77 (1989).

${ }^{39}$ S. Nojima, J. Appl. Phys. 58, 3485 (1985).

${ }^{40}$ Y. Mochizuki and T. Ikoma, Jpn. J. Appl. Phys. 24, L895 (1985).

${ }^{41}$ J. R. Morante, J. Samitier, A. Pérez, H. Altelarrea, and S. Gourrier, J. Appl. Phys. 60, 1661 (1986).

${ }^{42}$ H. J. von Bardeleben, N. T. Bagraev, and J. C. Bourgoin, Appl. Phys. Lett. 51, 1451 (1987). 